\title{
Llaqta/ciudad: espacio de tensión social en la poesía que- chua de Kusi Paukar
}

Llaqta/city: space of social tension in the quechua poetry of Kusi Paukar

\author{
Óscar Huamán Águila' \\ Universidad Nacional Mayor de San Marcos, Lima, Perú \\ Equipo de Investigación Ima kilqasta taki: IIH, FLCH-UNMSM² \\ oscar_huag@hotmail.com
}

\section{RESUMEN}

En el presente trabajo nos proponemos aproximarnos a la poesía quechua escrita, de manera específica al poemario Sonqup Jarawiinin, Umapa Jamutaynin, Runapa Kutipakuynin (1961), de Kusi Paukar (César Guardia Mayorga). En este texto la ciudad aparece como espacio de tensión social. La urbe ya no es del ayllu. El hombre andino siente que la comunidad humaniza, así como la ciudad deshumaniza, altera el orden y lo desconcierta. A ello se suma la existencia de dos grupos sociales disímiles (occidental y andino) en la urbe. El occidental es el que tiene pueblo, es decir, es dueño de la ciudad. Esto ha producido un desequilibrio en llaqta en la ciudad. En el presente trabajo nos proponemos examinar estos aspectos y vincularlos a la percepción

1 Estudiante de pregrado de Literatura de la Universidad Nacional Mayor de San Marcos. Ha participado como ponente en el IV, V y VI Coloquio de Lengua y Cultura en los Andes, en 2013, 2014 y 2015, respectivamente, actividades realizadas por la Cátedra de Lengua Quechua. Asimismo, ha participado en la XVI Jornada Andina de Literatura Latinoamericana de Estudiantes, "Ciudades escritas: invención y transformaciones de Latinoamérica en la Literatura», actividad organizada por Universidad Nacional de Bogotá, Colombia; en las XV Jornadas Andinas de Literatura Latinoamericana para Estudiantes (JALLA-E) Santiago 2014, organizadas por la Universidad de Santiago de Chile; y en el V Encuentro de Jóvenes Investigadores en Literatura Peruana de 2014, organizado por el Instituto de Investigación Humanísticas (IIH) de la Universidad Nacional Mayor de San Marcos. Ha intervenido en 2013, 2014 y 2015 , en condición de colaborador, en los proyectos de investigación del Instituto de Investigación Lingüística (Invel) y del IIH de la Universidad Nacional Mayor de San Marcos. Actualmente, participa como colaborador en los proyectos de investigación del IIH y del Invel.

2 Este trabajo forma parte del estudio «Ima qillqas taki? Tránsitos, voz y memoria en la poesía quechua contemporánea», proyecto de investigación que corresponde al periodo 20142015 del IIH de la Universidad Nacional Mayor de San Marcos, dirigido por el doctor Gonzalo Espino Relucé. Al mismo tiempo, corresponde al proyecto de tesis de licenciatura financiado por el Vicerrectorado de Investigación (VRI) de la Universidad Nacional Mayor de San Marcos para 2016. 
del hablante lírico andino y la configuración de la ciudad en la poesía quechua de la década de 1960. Resolveremos el tema propuesto desde la hermenéutica andina y con las categorías andinas, awqa, pacha y runa.

\section{PALABRAS CLAVES}

Llaqta, runa, poesía quechua, yo lírico andino, tensión social

\section{ABSTRACT}

This work has the purpose of approaching to the writing quechua poetry, specifically to Sonqup Jarawiinin, Umapa Jamutaynin, Runapa Kutipakuynin (1961) by Kusi Paukar (César Guardia Mayorga). In this text, the city appears as a space of social tension. The town is not the ayIlu yet. The Andean man feels that the community turns into more human, while the city loses its condition of humanity, alters the order and disconcerts him. In addition, there exist two very different social groups (Western and Andean). The Western group is which has town, in other words, it is the owner of the city. This has produced a disequilibrium in the llaqta/ the city. We are going to examine this change to suggest a link between it and the perception of the Andean lyric speaker; also, we are going to study the city's configuration in the quechua poetry of the decade of the 1960's. In the XX contery for twes reason, we are going to solve the topic proposed from of the Andean hermeneutic and the Andean categories of awqa, pacha and runa.

\section{KEYWORDS}

Llaqta, runa, quechua poetry, Andean lyric l, social tension

América Latina es un continente que alberga múltiples culturas desde tiempos inmemoriales. Aquellos pueblos perviven hasta nuestros días. A este espacio también llegaron otras culturas procedentes de otras latitudes; en este caso, la cultura occidental, que se ha impuesto sobre los pueblos que existían en esta área, con sus modos de pensar y su tecnología. Este suceso nos lleva a preguntarnos sobre la percepción de los pueblos indígenas en torno a los espacios que construyeron los occidentales, como ciudades, caminos, puertos, entre otros, lo que configura como nuevo escenario. En el siglo XX varían las percepciones de los quechuas, 
en este caso en la poesía escrita de los pueblos indígenas. Sobre este aspecto nos compete hurgar desde el campo literario.

A mitad del siglo XX empiezan las publicaciones de algunos indígenas o mestizos en su propia lengua. De manera específica en el campo de la poesía, se han publicado obras de carácter monolingüe y bilingüe, o sea, como menciona Espino (2012) en «la palabra florida...» (p. 370). El dominio de la tecnología de la escritura les ha permitido el ingreso al mundo letrado. Deben considerarse los escritos de Kusi Paukar en la cultura quechua del Perú, Irma Pineda en la cultura zapoteca de México, Elicura Chihuailaf en la cultura mapuche de Chile, Hugo Jamioy en la cultura camëntsá de Colombia, Susy Delgado en la cultura guaraní de Paraguay y Briceidad Cueva en la cultura maya en Guatemala. Estos son algunos de los poetas indígenas de las culturas amerindias que han contribuido a la conformación de una literatura indígena de América Latina.

En esta vertiente literaria se encuentra la poesía quechua, y dentro de ella el poemario Sonqup Jarawiinin, Umapa Jamutaynin, Runap Kutipakuynin (1961) [El canto del corazón, El pensamiento de la cabeza, La respuesta del hombre] ${ }^{3}$, de Kusi Paucar, seudónimo de César Guardia Mayorga. Este poemario fue publicado en Lima en 1961 como texto monolingüe; es decir, está escrito solo en lengua quechua chanka. Recién en 1975 apareció una edición bilingüe.

En la poesía quechua de César Guardia Mayorga la ciudad se configura como un espacio de tensión social de cultura disímil de Occidente, que deshumaniza al hombre quechua. Esto se debe, desde nuestra óptica, a que se imponen los valores culturales occidentales en la metrópoli; inclusive el espacio del tiempo moderno está diseñado para las sociedades occidentales y no para los pueblos indígenas. Por ello, no se puede dar el encuentro entre estas dos matrices culturales diferentes. $Y$ esto tiene una consecuencia negativa para el runa, porque no pueden enraizarse sus valores culturales y además son ajenos a la cultura de Occidente. Este acontecimiento que se encadena en la urbe - como veremos más adelante-desconcierta al runa.

En términos poéticos, la crisis cultural del hombre andino invade la memoria que se reactualiza, para poder subvertir el mundo contemporáneo y no para añorar, de manera nostálgica, el tiempo del runa:

Ñaupa pachakunapi

Kusilla kausarqanchik,

Llaqtanchikta kuyaspa,
En los tiempos antiguos vivíamos felices,

Amando a nuestro pueblo,

3 Las traducciones de la poesía quechua de César Guardia Mayorga son nuestras. 
$[\ldots .$.

Runa masinchikta yanapaspa. (Kusi Paukar, 1961, p. 27).
[...]

Apoyando a nuestro congéneres.

(La traducción es nuestra).

En aquel tiempo, del runa, el ser humano podía vivir de manera armónica con los demás seres que ocupaban el espacio. El lugar no solo era para ñuqanchik («nosotros») y ñuqayku («nosotros»), sino también para los demás que se encontraban en el universo y las demás culturas. No era privativo de un individuo o un grupo social. Esto se desprende de los pronombres mencionados por el sujeto enunciador. En lengua quechua estos pronombres plurales no tienen el mismo nivel semántico. El ñuqanchik incluye a todos; en cambio, el ñuqayku excluye a otros. Como se puede apreciar en la cita, entre ellos, ñuqanchik y ñuqayku, existía una armonía social.

Es decir, en el tiempo andino el ayllu se desenvolvía en un estado de equilibrio; porque el runa regía sus valores culturales (la solidaridad, la generosidad, la religiosidad, la relación con la tierra y la reciprocidad) en el universo andino. Existía una relación entre el runa y el espacio físico y el runa masininchik («nuestros congéneres»). Esto no se encuentra de manera explícita en los versos de César Guardia Mayor, pero se deduce. Porque, en el mundo andino, para que el sujeto pueda alcanzar la condición de humanidad tiene que cumplir los valores y las normas del ayllu. Además, requiere al otro para su determinación. Al respecto, Pablo Landeo señala: «Ser runa convoca la presencia del otro, del runamasi (gente como yo), porque el sujeto ñuqa se humaniza en cuanto se reconoce en el otro y a su vez es reconocido» (2012, p. 59). En el verso se menciona la presencia de otros en el espacio y en el tiempo del mundo quechua, en la misma condición del runa; pero esto no se produce en el tiempo moderno, sino más bien el ser humano se ha individualizado. Y el espacio es ajeno al runa. Solo un individuo es dueño del espacio, goza de poder y de bienestar material:
Wiraqochallam kunanqa wiraqucha, Solo el señor es ahora señor, Wiraqochallam llaqtayoq, Solo el señor tiene ciudad, Wiraqochallam qollqe qoriyuq, Solo el señor tiene plata de oro, Wiraquchallam kusikun. (Kusi Paukar, 1961, p. 29). Solo el señor se regocija. (La traducción es nuestra).

La ciudad es la propiedad de un individuo en el tiempo moderno. En la voz lírica se configura el nuevo señor como un individuo que no carece de nada, porque tiene bajo su dominio al espacio y también a él pertenece el dinero forjado de un metal valioso; en otras palabras, es propietario de los bienes. Además, el lugar común se configura como un objeto que un sujeto o grupo puede apropiarse, pero en desmedro de los demás seres 
humanos. El runa está privado de pertenencia a la urbe y no accede al de poder político ni al bienestar social.

Hay cuatro elementos, en los versos citados, que se requieren subrayar: wiraqucha (el señor), la ciudad, el dinero y, por último, la felicidad. En primer lugar, se menciona a una persona individual y no colectiva; en otras palabras a un sujeto individualizado. Este adquiere un poder más grande que un señor que existía en el tiempo andino. El dueño de la urbe ha traspasado el límite de poder que conoce el yo lírico andino. En segundo lugar, la urbe y el dinero es pertenencia de este nuevo sujeto que tiene poder. Estos dos objetos corresponden al campo material, ya que son producto de la intervención de la mano del hombre. En tercer lugar, la felicidad, que tiene ver con el estado de ánimo del ser humano. Pero ello está relacionado con el estado material, como se puede apreciar en los versos. Por ese motivo, el nuevo señor del tiempo moderno puede acceder por el poder que emana hacia ser constancia.

Estos elementos mencionados en la poesía quechua, como la ciudad y el dinero, hacen referencia a una sociedad individualista y mercantilista. Además, la moneda tiene un carácter simbólico en el poema. Es un metal de alta estimación de una sociedad materialista, y tiene un carácter cuantitativo y un poder que puede remplazar sin ninguna dificulta a cualquier objeto en un mundo simbólico. El sujeto que posee este también tiene poder sobre los demás y puede alcanzar la felicidad en el mundo material.

La cultura occidental no solo es propietaria de la urbe, sino también ha impuesto sus normas, sus leyes y los valores culturales. En cambio, el runa no ha quedado al margen. A estas alturas de nuestro análisis, cabe mencionar la incompatibilidad de las prácticas culturales de los pueblos indígenas que habitan en este continente desde los tiempos inmemoriales con respecto a los foráneos, venidos de otro espacio. Esto ha generado en la ciudad una confrontación de dos fuerzas antagónicas. No solo se ha producido el desequilibrio de poder, la desigualdad social, sino también se ha trastocado el orden cultural del hombre andino:

Jallpayoq, mana jallpayoq, El que tiene tierra, ya no tiene tierra, Llaqtayoq, mana llaqtayoq, El que tiene pueblo, ya no tiene pueblo, Tukuy imayniyoq, El que tiene todo, Mana imayoq rikukun. Se encuentra sin nada. (Kusi Paukar, 1961, p. 23). (La traducción es nuestra).

En el poema la urbe es configurada como un espacio ajena al hombre del andino y esto se ha generalizado, ya que ha pasado al otro ámbito espacial; en este caso, a la tierra. Ello ha generado un desequilibrio al runa y una orfandad material. Por ende, aquello que le correspondía ya no le pertenece; en otras palabras, han sido desplazados de su pertenencia por 
aquellos sujetos que no pertenecen al ayllu. En esta configuración de la tragedia del pueblo quechua es donde la voz lírica adquiere un carácter colectivo, porque enuncia las desgracias de un pueblo y no de un individuo, a pesar de que no se incluye dentro de los desamparados. Además, su discurso constituye una voz de denuncia de lo que acontece en la urbe y en el universo con el sujeto andino.

El territorio para las culturas andinas y amazónicas tiene un significado distinto que para el occidental (hispano). En la concepción de los pueblos indígenas, el espacio tiene una connotación de vida; por tal sentido, se concibe como base para la existencia humana, cultural y también para otros seres vivientes que habitan en ella. En un estudio realizado por Teleandes Producciones, se menciona que: «la tierra y sus recursos no están desligados, sino hay una integración, es decir, con el mundo social, económico y emocional. Ya que es la base para su bienestar físico y espiritual, y, es más, es el sustento de su identidad y el lazo con el pasado y el futuro» (2009, p. 20). La ruptura de una de estas relaciones en el mundo andino es negativa, ya que pone en peligro su existencia en el universo. En el poema citado de César Guardia Mayorga se ha invertido esta relación. El pueblo indígena ha perdido todo.

Entonces estamos ante un tiempo de oscuridad, ya que el orden social del runa se ha invertido en el mundo andino. Esto se hace evidente en la pérdida de la tierra y por la ajenidad de la urbe en el tiempo contemporáneo. Para la cultura quechua, la territorialidad posee un significado complejo, ya que no es un simple espacio que uno puede apropiarse y explotar. Para esta cultura, es un lugar que sostiene la vida y, desde luego, existe en todo lo que ocupa espacio. Además, en ella se encuentran sus dioses y el alma de sus antepasados. Cada uno de ellos cumple un rol social y existencial en la concepción del mundo andino.

Sin embargo, en el tiempo occidental todo esto se ha invertido; en otras palabras, se ha desvanecido. En la voz del yo lírico andino, el territorio y la ciudad son ajenos a la cultura quechua que sigue perviviendo en el tiempo. Además, el espacio es deshumanizante para el runa.

La urbe es un espacio que no posibilita el desarrollo comunitario del runa, ya que los valores culturas de los foráneos se han afincado y son incompatibles con los pueblos autóctonos. Para González Gómez (2014), la ciudad del tiempo moderno está pensada y estructurada desde la percepción individual (p. 74). Ello supone que las prácticas andinas son de carácter colectivo. Y, por ende, no permite el desarrollar de los valores culturales colectivos del mundo quechua. Este y otros aspectos sociales que se manifiestan en la ciudad conllevan a la deshumanización del hombre del Ande, ya que sus valores culturales se han debilitado ante los valores 
occidentales. La solidaridad y la cooperación con los demás congéneres no existen. A esto se suma el carácter de insensibilidad del sujeto quechua que vive en el tiempo moderno. Los fenómenos sociales que conmueven al mundo no les importan, ni mucho menos su condición de ser wakcha.

La insensibilidad y la indiferencia reinan en la urbe a pesar de que se encuentra - el runa - en un tránsito de un estado a otro estado:

\begin{tabular}{|c|c|}
\hline Punchau llantuyachkanña, & El día está ensombreciendo, \\
\hline$[\ldots]$ & {$[\ldots]$} \\
\hline Manataq pipas & Pero ninguno \\
\hline Kaymi, kaypim, chaynam, & Esto es, aquí está, así es \\
\hline Nispa niwanchu. & No me dicen, \\
\hline Ruranaymi, ruranayn & Tengo que hacer, $t$ \\
\hline Ñampa apasqan, & Llevado por el camino, \\
\hline $\begin{array}{l}\text { Nampi chinkarparin. } \\
\text { (Kusi Paukar, 1961, p. 23). }\end{array}$ & $\begin{array}{l}\text { En el camino desaparecen. } \\
\text { (La traducción es nuestra). }\end{array}$ \\
\hline
\end{tabular}

El hablante lírico percibe que el hombre del tiempo moderno es indiferente e individualista ante el mundo y los demás seres humanos. Su trabajo está sobre los demás aspectos sociales que hace que sea un runa. El sentido colectivo y la pertenencia a una comunidad no existen para el sujeto de la urbe, porque el quehacer cotidiano es lo más importante para él, más que sensibilizarse con los aspectos sociales que se dinamizan en la sociedad, a pesar de que se encuentra en un tiempo liminal.

El quehacer cotidiano del sujeto urbano es agotador. Por ello, busca un momento de descanso para escapar del mundo que lo tortura y luego para continuar en ese mundo que le succiona la fuerza. Y, es más, es condenado a la muerte simbólica, ya que no queda nada de él en el mundo.

Como se puede percibir en las estrofas citadas de Kusi Paucar, el sujeto enunciador verbaliza lo que acontece en la ciudad; yo diría que verbaliza lo que sucede en el espacio continental de americano. No solo la cultura quechua ha sufrido los embates del mundo occidental, sino también las otras culturas amerindias. En los versos se manifiestan los fenómenos sociales que afectan al runa y no al occidental; es decir, el discurso gira en torno al sujeto andino. Asimismo, se muestran dos elementos muy importantes que nos permitirían comprender la visión del runa que percibe los hechos y sobre los sujetos que viven en la ciudad. En primer lugar, la naturaleza aparece como un signo y tiene una función metafórica, ya que anuncia la aproximación de un tiempo determinado (de oscuridad). La oscuridad es un signo para el yo lírico andino, porque en ella encuentra la manifestación de un acontecimiento social que está por llegar. Sin embargo, no hay una certeza, en el conocimiento del poeta, al respecto de que es positivo o negativo para la cultura andina. Esto crea un 
desconcierto en la concepción del runa; por un lado, es un signo (el oscurecer) $y$, por otro lado, no lo es, porque genera la incertidumbre. A estas alturas de nuestro análisis, podemos decir que en el tiempo moderno los signos que permitían comunicar al hombre quechua han dejado de ser signos. En otras palabras, en la urbe la naturaleza ya no cumple la función de ser signo. El otro elemento que se configura en el poema citado es el camino. Este elemento, igual que el anterior, cumple una función de metáfora dentro del enunciado; conduce al hombre y no un automóvil u otro medio de trasporte que existe en la ciudad, pero no lo es. El sujeto que se encuentra en el trayecto no llega al final ni mucho menos deja signo de su existencia en el mundo.

En segundo lugar, expone la existencia de dos clases de sujeto: insensible y sensible. Para el primero, el fenómeno social que se desarrolla y que se aproxima no le causa extrañeza, pese a que lo oprime su condición de ser humano. Dentro de ellos se encuentra el hombre quechua, porque la voz del enunciador está dirigida hacia un runa. En otras palabras, el dialogo es entre los hombres del Ande. El sujeto ha perdido sus valores culturales que hace que sea hombre; esto es, ha dejado de ser un sujeto de la relacionalidad.

La pérdida de los valores culturales del mundo quechua es negativa para el hablante lírico andino, porque deja de ser runa; en consecuencia, será nefasta para la existencia del sujeto colectividad. La razón es sencilla: porque pierde la condición de ser eterno en el espacio y el tiempo.

El runa ha perdido su condición de corporeidad y una existencia propia. Ahora es una sombra y depende de otro; en este caso, de la luz y de la existencia ajeno a él. En otras palabras, constituye Ilantu («sombra»). En el primer verso, en la siguiente cita, se manifiesta este suceso. En el universo representado, todo lo que corresponde al mundo andino se encuentra en un estado de crisis. Sin embargo, en este mundo de caos social, cultural, no todo está perdido. Todavía hay una esperanza en el horizonte social, porque no todos (runakunas) han sido absorbidos por el tiempo deshumanizante del mundo occidental, como aquel que no ha ingresado al tiempo moderno: la sensibilidad, los valores culturales del mundo andino y la perceptibilidad de los acontecimientos sociales que afecta a la humanidad. Uno de ellos es el sujeto enunciador o, en otras palabras, el yo lírico andino; pero también están los demás runas. Este último no está de manera explícita en el poema, pero se deduce la enunciación de la voz lírica. El mensaje está direccionado a otro runa, no a un occidental (español). Por ello es que se enuncia los acontecimientos sociales que se manifiestan en la urbe: 


\section{Punchau tuyachkanña, El día está oscureciendo, \\ [...] [...] \\ Runakunañataq Ilantu hina Los hombres ya también como la sombra Suntuykachaspa, Se apilonan, \\ Samanaymi, samanaymi, nispa, Tengo que descansar, tengo que descansar, diciendo, Ñampa apasqan, Llevado por el camino, Tutayaqpi chinkarparin. En la oscuridad desaparece. (Kusi Paukar, 1961, p. 23). (La traducción es nuestra).}

En las estrofas se muestra que el runa se ha reducido a un solo aspecto de la vida; es decir, solo al campo laboral. Los demás factores que permitían su desarrollo social y existencia individual y cultural ya no son un acto ético. Más bien lo que prima es la individualidad y la indiferencia. Esto se hace visible en la voz del sujeto enunciador. Las estructuras sociales del ayllu andino se han desintegrado.

Pablo Landeo (2012) menciona que, para adquirir la condición del runa, en el mundo andino hay normas sociales que se acatan, se reconocen y se practican. Además, esto implica pertenecer a un territorio, tener un origen común, hablar el mismo idioma y compartir las tradiciones del pueblo (p. 60). En este mismo sentido señala González: «la participación colectiva o la vida en comunidad es la base, porque en ella va uno aprendiendo distintas formas de proceder, principios, comportamientos, y valores, sobre todo, y cada uno de ellos te configura, te dan elementos para proceder, en la vida en su conjunto» (2014, p. 78) (las cursiva son nuestras). Entonces, para que logre la condición de ser runa, el sujeto debe cumplir varias prácticas culturales que existen en la comunidad. Sin embargo, esto no es posible en llaqta («ciudad») del tiempo moderno, como se puede percibir en la poesía de quechua que analizamos. Los valores culturales del mundo andino que regían su universo se han perturbado.

La pérdida del sentido de la relacionalidad, de la reciprocidad, de la sensibilidad del runa, se convierte en un fenómeno desconcertador para el yo lírico andino. Porque nadie logra develar el acontecimiento que ocurre en la suciedad en la que se encuentran. Esto lo veremos en la cita que viene. Y también debo mencionar el otro aspecto que coadyuva para que se sienta: el sujeto quechua, desconcertado en el espacio que ocupa (en la metrópoli); con esto me refiero a la no pertenencia a la cultura que predomina y a la urbe.

La individualización y la indiferencia que predominan en el tiempo moderno no solo afectan a aquellos que fueron absorbidos por los valores occidentales, sino también a las personas que no han sido engullidas por la sociedad deshumanizante. Esto se hace evidente en el desvanecimiento del sujeto enunciar y los demás individuos. Los dos están en la misma 
condición de extensión, porque no solo desaparecen aquellos que han perdido los valores culturales del mundo quechua, sino también el otro que mantiene y práctica.

$\begin{array}{ll}\text { Jinamanta, ñoqapas, } & \text { Asimismo, yo también, } \\ \text { Sapallayña rikukuspa, } & \text { Al encontrarme solo, } \\ \text { Upay upa tutayaypi } & \text { En la inmensa oscuridad } \\ \text { Chinkarparini, } & \text { Desaparezco, } \\ \text { Manaraqpas imatapas } & \text { Sin saber todavía } \\ \text { Yachachkaspa. } & \text { Algo. } \\ \text { (Kusi Paukar, 1961, p. 23). } & \text { (La traducción es nuestra). }\end{array}$

La desaparición tanto de la colectividad como del individuo hace que el yo lírico andino despliegue una mirada autorreflexiva. En el poema «Tapukuni» («Me pregunto») se interroga sobre lo que acontece en su comunidad y en su mundo interno: « ¿Maymantam jamun sonquyman / Kay chinka ñampi / Wayta tarii munay?» (Kusi Paucar, 1961, p. 23) [De dónde proviene a mi corazón / En este camino dificultoso / El deseo de encontrar la flor]. Como se puede apreciar en estas estrofas, hay un desdoblamiento del sujeto lírico hacia su mundo interno. Allí trata de encontrar la procedencia de esa preocupación que lo inquieta. El yo lírico andino no solo busca la explicación del desequilibrio que se ha producido en la urbe, en lo externo, sino también en su ámbito interno.

Hay una preocupación en el yo poético por encontrar el origen del mal que aqueja al runa y también el bienestar de ellos. Lo que hemos planteado se desprende de la dualidad configurada en los versos que analizamos. La espina y la flor son opuestos a pesar de que pertenecen al mismo género. El primer elemento tiene que ver más con la incomodidad que causa a un cuerpo; en cambio, el segundo con la tranquilidad que causa en la sensibilidad del sujeto.

Para el runa, la metrópoli es un estado de caos social y cultural, porque todo se encuentra alterado: el camino ha perdido su sentido de ser un medio que permite la seguridad al hombre que viaja, el ayllu ha perdido sus valores culturales y se encuentra desconcertado.

Por un lado, esto nos permite determinar la importancia de la identidad y los valores culturales determinan una condición social. Por otro lado, también nos permite preguntarnos: ¿qué determina y hace que sea considerado como un sujeto andino un individuo que vive en la urbe? Como podemos percibir en el análisis del poemario, la ciudad no permite desarrollar los valores culturales del runa, no lo deja enraizarse. Pero esto tiene una consecuencia negativa para el mundo quechua, ya que conduce a la muerte de la colectividad y, por último, del individuo. 
En conclusión, la urbe es un espacio de encuentros y de desencuentros entre dos matrices culturales disímiles (por un lado, la cultura andina $y$, por el otro, la cultura occidental). Además, constituye un punto clave para aniquilar las leyes, éticas y normas sociales del mundo andino. La ciudad deshumaniza al runa por ser un espacio que no permite practicar los valores culturales del mundo quechua. En la poesía quechua de César Guardia Mayor se manifiesta el tiempo mítico y utópico del mundo andino. 


\section{REFERENCIAS BIBLIOGRÁFICAS}

Barquero, J. (1980). La poesía quechua actual en el Perú. Tarea, 3, pp. 39-40.

Berman, M. (1999). Todo lo solido se desvanece en el aire. La experiencia de la modernidad. Madrid: Siglo Veintiuno Editores.

Chihualaf, N. E. (2010). Un oralitor que habla con la palabra de sus abuelos. Espíritu de pájaro en pozos del ensueño. Bogotá: Ministerio de Cultura.

Cornejo Polar, A. (1994). Escribir en el aire: ensayo sobre la heterogeneidad socio-cultural en las literaturas andinas. Lima: Horizonte.

Espino Relucé, G. (2012). Ñuqanchis takikuna: marcas andinas -ancestrales- en la poesía y en la narrativa moderna. Escritura y Pensamiento, 31, pp. 121-140.

(2012). Tukurita nunacay pisccomanta. Lectura desde el otro lado de Latinoamérica, representación de la poesía quechua de Wiñay Mallki. Actas del Congreso Internacional de «Poesía hispanoamericana de la vanguardia a la posmodernidad» (pp. 369-386). Lima: Editorial San Marcos.

Estermann, J. (2006). Filosofía andina. Sabiduría indígena para un mundo nuevo. La Paz: Edoba.

González Gómez, A. (2014). Cosmovisión indígena contemporánea. Hacia una descolonización del pensamiento. México D. F.: Visión Impresa.

Huamán López, C. (2015). Urpischallay: Tradiciones poéticas, memoria y cultura popular andina en el wayno. Lima: Altazor, Universidad Nacional Autónoma de México y Universidad Nacional Mayor de San Marcos.

Kusi Pauka (1961). Sonqup jarawiini, umapa jamutaynin, runap kutipakuynin. Lima: Minerva.

Landeo Muñoz, P. (2012). Categorías andinas para una aproximación al willakuy umallanchikpi (Seres imaginarios del mundo andino) (tesis de maestría en Literatura). Universidad Nacional Mayor de San Marcos, Lima.

Llanque Chana, D. (1969). Los valores culturales de los aymaras. Allpanchis, 1, pp. 123-133.

Mamani Macedo, M. (2012). Quechumara. Proyecto estético-ideológico de Gamaliel Churata. Lima: Fondo Editorial de la Universidad de Ciencias y Humanidades.

Noriega Bernuy, J. (2012). Caminan los apus. Escritura andina en migración. Lima: Pakarina-Knox College. 
Teleandes Producciones. (2009). Los achuar en la cuenca del río Pastaza / The Achuar in the Pastaza River Basin. Lima: Garfieril.

Recibido: mayo de 2016

Aceptado: julio de 2016 